\title{
Ordem do treinamento com pesos, capacidade funcional e carga de treino em idosos treinados: ensaio clínico aleatorizado
}

\author{
Order of resistance training, functional capacity and training load in \\ trained elderly: randomized clinical trial
}

\author{
Fábio Luiz Cheche Pina ; Edilaine Fungari Cavalcanteㄹ; Crisieli Maria Tomeleri²; \\ Matheus Amarante do Nascimento2,3; Aline Mendes Gerage 2,4; Renata Selvatici Borges Januório'; \\ Arli Ramos de Oliveira ${ }^{5}$; Edilson Serpeloni Cyrino²

\footnotetext{
1 Grupo de Estudo e Pesquisa em Atividades de Academia. Centro de Pesquisa em Ciências da Saúde. Universidade Norte do Paraná - UNOPAR. Londrina, PR - Brasil

2 Grupo de Estudo e Pesquisa em Metabolismo, Nutrição e Exercício. Centro de Educação Física e Esporte. Universidade Estadual de Londrina - UEL. Londrina, PR - Brasil.

3 Faculdade Estadual de Educação, Ciências e Letras de Paranavaí. Universidade Estadual do Paraná - UNESPAR. Paranavaí, PR -

3 Faculdade
Brasil.

4 Centro de Desportos. Universidade Federal de Santa Catarina - UFSC. Florianópolis, SC - Brasil.

5 Centro de Educação Física e Esporte. Universidade Estadual de Londrina - UEL. Londrina, PR - Brasil.
}

\section{Endereço para correspondência:}

Fábio Luiz Cheche Pina

Av. Maringá, 478 - Apto 106

86060-000 - Londrina, PR [Brasil]

fabiocheche@hotmail.com

\section{Resumo}

Introdução: a ordem do treinamento com pesos (TP) pode estar relacionada aos benefícios observados nesta modalidade. Objetivo: avaliar a ordem de TP na capacidade funcional e carga de treino. Métodos: dezoito idosos treinados foram aleatorizados: sequência $\mathrm{A}$ (SEQ A), dos grandes para os pequenos grupos musculares e sequência B (SEQ B), ordem inversa a SEQ A. A flexibilidade, tarefas funcionais, resistência muscular e carga de treino foram verificados. Resultados: interação foi observada para a flexibilidade, em que a SEQ B (+ 18,7\%) apresentou resultado significativo, quando comparada à SEQ A (- 18,4\%). Efeito do tempo foi encontrado para tarefa funcional (SEQ A $=-2,9 \%$ e SEQ B $=-1,9 \%$ ), resistência muscular (SEQ A $=+11,5 \%$ e SEQ B $=+8,9 \%$ ) e carga de treino (SEO A $=+54,0 \%$ e SEQ B $=+74,3 \%$ ). Conclusão: TP é efetivo para a melhora da tarefa funcional, da resistência muscular e da carga de treino, porém a ordem pode influenciar na flexibilidade.

Descritores: Treinamento de Resistência; Autonomia Pessoal; Esforço Físico.

\begin{abstract}
Introduction: The order of resistance training (RT) may be related to the benefits observed in this modality. Objective: To evaluate the order of RT in functional capacity and training load. Methods: Eighteen trained elderly were randomized: sequence A (SEQ A), from large to small muscle groups and sequence B (SEQ B), reverse order to SEQ A. Flexibility, functional tasks, muscle endurance and training load were verified. Results: Interaction was observed for flexibility, where the SEQ B (+ 18.7\%) presented significant result when compared to SEQ A $(-18.4 \%)$. Effect of time was found for functional task (SEQ A $=-2.9 \%$ and SEQ B $=-1.9 \%$ ), muscle endurance (SEQ A $=+11.5 \%$ and SEQ B $=+8.9 \%$ ) and training load (SEQ A $=+54.0 \%$ and SEQ B $=+74.3 \%$ ). Conclusion: $\mathrm{RT}$ is effective for improving functional task, muscular endurance and training load, but order can influence flexibility.
\end{abstract}

Keywords: Resistance Training; Personal Autonomy; Physical Exertion. 


\section{Introdução}

Uma das principais mudanças observadas no envelhecimento é o declínio na capacidade funcional, ocasionando perda gradual da autonomia e, consequentemente, diminuição da qualidade de vida ${ }^{1}$. Considerando que a adoção de um estilo de vida fisicamente ativo pode minimizar muitas perdas observadas com o avançar da idade, o treinamento com pesos (TP) tem sido amplamente recomendada para os ido$\operatorname{sos}^{1-2}$, por ser uma estratégia não farmacológica, que pode gerar importantes benefícios à saúde, como o aumento da força e da resistência muscular $^{3}$, acréscimo da flexibilidade ${ }^{4}$, melhora no desempenho funcional ${ }^{5}$, diminuição do risco de quedas ${ }^{6}$, além de prevenir o desenvolvimento de inúmeras doenças crônico-degenerativas ${ }^{1,2,7}$.

Grande parte dos beneficios associados aos programas de TP são protocolo-dependentes ${ }^{8}$. Dessa forma, é necessário a manipulação adequada do volume (número de exercícios, séries e repetições; duração e frequência ao treinamento) e da intensidade (carga levantada, velocidade de execução, intervalos de recuperação entre as séries) ${ }^{8}$. Dentre as variáveis do TP que podem ser manipuladas, a ordem de execução dos exercícios influencia tanto o volume quanto a intensidade durante as sessões, colocando em risco a efetividade dos programas de treinamento ${ }^{9,10}$. Apesar disso, verifica-se na literatura que as poucas investigações sobre o impacto desta variável foram conduzidas, predominantemente, em estudos com delineamento transversal ${ }^{9-12}$ e em adultos jovens ${ }^{9,13-15}$, o que limita a compreensão das respostas adaptativas em idosos treinados.

Um programa de TP que inicia pelos grandes grupos musculares, seguindo para os pequenos grupos tem sido a proposta realizada por muitos profissionais e, também, a recomendada no Posicionamento do American College of Sports and Medicine ${ }^{8}$ quanto à ordem de execução dos exercícios. Tal indicação está baseada na premissa que, se os grandes grupos musculares forem treinados no início, maior resistência poderá ser empregada, visto que os músculos estão descansados e, portanto, suportam maior sobrecarga. Porém, esta é uma das variáveis menos estudadas na população idosa, especialmente, em idosos com experiência em TP.

Existem na literatura quatro estudos com a população idosa ${ }^{10,12,16,17}$, os quais limitaram a análise do número de repetições executadas, $\mathrm{VO}_{2 \text { máximo }}$ e a percepção de esforço por meio de um delineamento transversal ${ }^{10,12,17}$ e ao acompanhamento (efeito crônico) da composição corporal ${ }^{16}$. Sendo a capacidade funcional determinante na saúde do idoso ${ }^{5,18}$, e a progressão na carga de treino uma estratégia de análise para a evolução da sobrecarga do treino ${ }^{4}$, a procura por informações a partir de delineamentos longitudinais pode contribuir para a determinação da ordem de posicionamento dos exercícios, especialmente em idosos treinados ${ }^{19}$, de modo que as respostas adaptativas possam ser mais favoráveis, beneficiando a aderência ${ }^{20}$ dos mesmos.

Uma vez que a ordem pode estar relacionada com respostas observadas na prática do treinamento ${ }^{9}$, o objetivo do presente estudo foi avaliar a influência da ordem de execução do TP na capacidade funcional e progressão da carga de treino em idosos treinados. A hipótese é que o programa de TP, na ordem dos grandes para os pequenos grupos musculares, favoreça maior carga de treino e, como consequência, melhora na capacidade funcional.

\section{Métodos}

\section{Delineamento experimental}

Ensaio clínico aleatorizado foi desenvolvido a partir do banco de dados produzido pelo projeto: "O treinamento com pesos para indivíduos acima de 50 anos". O experimento teve duração de onze semanas, sendo dividido em dois momentos. $\mathrm{O}$ pré-treinamento (baseline $=$ duas semanas) correspondeu à seleção da amostra, seguido de entrevistas com a aplicação de questionários (IPAQ e Mini Mental) e avaliações (capacidade funcional e antropometria). Na sequência, os participantes foram aleatorizados de maneira 
balanceada (random.org), em dois grupos, de acordo com a ordem de treinamento. A sequência "A" realizou o TP dos grandes para os pequenos grupos musculares (SEQ A, $70 \pm 5$ anos, 73,4 \pm $14,5 \mathrm{~kg}$ e $1,64 \pm 0,06 \mathrm{~m}$ ) na seguinte ordem: supino em banco horizontal com predominância do músculo peitoral (SH), puxada articulada com predominância dos músculos dorsais (PA), rosca bíceps na barra com predominância do músculo bíceps (RB), tríceps no pulley com predominância do músculo tríceps (TPU), cadeira extensora com predominância do músculo quadríceps (CE), mesa flexora com predominância dos músculos posteriores de coxa (MF), cadeira abdutora com predominância dos músculos glúteo médio e mínimo $(\mathrm{CAB})$ e cadeira adutora com predominância dos músculos adutores (CAD). Para a sequência "B" (SEQ B, $69 \pm 6$ anos, 69,8 \pm 11,4 kg e 1,64 $\pm 0,05 \mathrm{~m}$ ) se adotou a ordem dos pequenos para os grandes grupos musculares: TPU, RB, PA, SH, $\mathrm{CAD}, \mathrm{CAB}, \mathrm{MF}$ e $\mathrm{CE}^{9-17}$. A montagem do programa de treinamento obedeceu à ordem alternada por articulação (membros superiores inicialmente, finalizando com membros inferiores), diferenciando entre as sequências a ordem de execução dos exercícios. Por fim, após sete semanas de intervenção, no pós-treinamento (duas semanas), foram aplicadas as reavaliações nas capacidades funcionais. Avaliações da capacidade funcional (flexibilidade, tarefa funcional e resistência muscular) foram realizadas na linha de base (prétreinamento) e na semana posterior às sete semanas de TP (pós-treinamento). Também, durante as sete semanas de intervenção, semanalmente a carga de treino foi registrada.

\section{Participantes}

Foram selecionados 18 homens ativos fisicamente entre 60 e 80 anos com experiência em TP. O tamanho da amostra foi baseado em um alfa de $95 \%$, poder estatístico de $80 \%$ e uma perda amostral estimada em $20 \%$. Os idosos do presente experimento já faziam parte de um programa de $\mathrm{TP}$, nos últimos quatro meses anteriores ao início do estudo, visando resistência muscular (três sessões semanais, duas séries de 10-15 repetições máximas [RM], oito exercícios). Como critérios de inclusão, os selecionados não poderiam apresentar histórico de disfunções crônico-degenerativas não controladas e limitações musculoesqueléticas, que impedissem a prática do TP. O presente estudo seguiu os princípios éticos, sendo aprovado pelo Comitê de Ética em Pesquisa local (Processo: 21750) de acordo com as normas da Resolução 196/96 do Conselho Nacional de Saúde sobre pesquisas envolvendo seres humanos. Todos assinaram o Termo de Consentimento Livre e Esclarecido.

\section{Capacidade funcional}

Para a análise das capacidades físicas de calçar meias, de habilidades manuais e de se levantar do solo foram utilizadas as tarefas propostas por Andreotti e Okuma ${ }^{21}$. A flexibilidade de ombros, o teste de flexão do cotovelo e sentar e levantar de uma cadeira, bem como o teste Time Up and Go foram avaliados a partir do protocolo proposto por Rikli e Jones ${ }^{22}$. Para diagnóstico, a flexibilidade de ombro direito e esquerdo foram somadas para a análise da flexibilidade de ombro (cm). Da mesma forma, os testes de flexão do cotovelo unilateral por 30 segundos (lados direito e esquerdo) e os testes de sentar e levantar de uma cadeira em 30 segundos foram somados para a análise da resistência muscular total (rep.). Os testes de calçar meias, de se levantar do solo, de habilidades manuais e Time Up and Go foram somados para a análise da tarefa funcional total (seg.). Tanto o momento pré como pós-treinamentos foram realizados no mesmo período do dia (período da manhã) por um avaliador treinado, com erro técnico de medida menor do que $5 \%$ em cada tentativa.

\section{Carga de treino}

Visando reduzir a margem de erro, nas cargas utilizadas durante o TP, foram adotados os seguintes procedimentos: a) instrução padronizada fornecida antes dos treinamentos; 
b) conhecimento sobre a técnica de execução do exercício, durante as diferentes etapas de sua realização; c) posição adotada pelo idoso no momento do treinamento; d) adoção das seguintes etapas dos exercícios: posição inicial e desenvolvimento (fases concêntrica e excêntrica); e) estímulos verbais, a fim de manter o nível de estimulação durante os exercícios; e f) os pesos utilizados no estudo foram previamente aferidos em balança de precisão (Balmak, Laboratory Equipment Labstore, Curitiba, Paraná, Brasil).

Como os idosos eram habilitados para realizar 10 a 15 RM em suas sessões, os mesmos estavam adaptados a esta zona de treinamento. $\mathrm{O}$ incremento das cargas nas diferentes sessões variou entre 1 a $5 \mathrm{~kg}$. Em alguns casos foram utilizados pesos adicionais de $500 \mathrm{~g}$ para mais bem discriminar valores intermediários de cargas. Depois disso, a carga de treino para cada participante foi calculada semanalmente, utilizando a soma de carga empregada em todos os exercícios para cada semana.

\section{Protocolo de treinamento com pesos}

O protocolo foi estruturado nas recomendações vigentes para a prática de TP em ido$\operatorname{sos}^{2,23}$, sendo composto por oito exercícios, realizados com uma frequência de três sessões semanais (segundas, quartas e sextas-feiras) no período da manhã, totalizando 21 sessões para cada sequência (aderência de 94\%). Ambas as sequências foram executadas em duas séries de 10 a 15 RM com cargas fixas. O intervalo de recuperação entre as séries foi de 60 a 90 segundos, e entre os exercícios de dois a três minutos.

Vale destacar que as cargas utilizadas foram compatíveis com os intervalos de repetições estipulados para cada exercício, sendo determinadas após o desempenho de cada idoso nas sessões de familiarização (baseline) e ajustadas, individualmente, ao longo do período experimental, sempre que o limite superior de repetições predeterminadas para cada exercício fosse atingido, nas duas séries (15 RM), durante três sessões consecutivas. Assim, incrementos na or- dem de 2 a $5 \%$ para os exercícios de membros superiores e 5 a $10 \%$ para os exercícios de membros inferiores foram utilizados para que a intensidade inicial do treinamento fosse preservada ${ }^{8}$.

$\mathrm{Na}$ execução dos movimentos, os participantes foram orientados para inspirar na fase excêntrica e expirar na fase concêntrica, mantendo a velocidade de execução dos movimentos na razão 1:2 na fase concêntrica e excêntrica respectivamente $^{4,5}$. Todos os participantes foram orientados a manterem os níveis normais de atividade física e de hábitos alimentares, ao longo do estudo, e a não iniciarem novos programas de exercícios físicos ou dietas durante o período experimental.

Durante as sessões, um profissional e/ou estagiário de Educação Física realizou todo o acompanhamento, do início ao fim da sessão, controlando os intervalos de recuperação e ajustando os equipamentos para o uso dos participantes do estudo, bem como das funções tradicionais de manter a segurança e instruir a correta execução dos exercícios. Para isso, foi adotada como estratégia a manutenção de dois a três idosos por profissional e /ou estagiário de Educação Física.

\section{Tratamento estatístico}

O teste de Shapiro Wilk foi utilizado para a análise da distribuição dos dados e o teste de Mauchly a esfericidade. Análise de variância (ANOVA two-way) para medidas repetidas foi realizada para as comparações intra e intergrupos na capacidade funcional e carga de treino. O teste post hoc de Tukey foi empregado para a identificação das diferenças específicas nas variáveis, cujos valores de $\mathrm{F}$ encontrados foram superiores ao critério de significância estatística estabelecida $(p<0,05)$. Análise das modificações relativas individuais (responsividade ao treinamento) foi calculada através do delta percentual (pós-treinamento x 100/pré-treinamento) - 100. Para análise dos dados foi utilizado o pacote STATISTICA para Windows, versão 10.0 (StatSoft Inc, Tulsa, OK, USA). A magnitude das diferenças foi calculada a partir do tamanho do efeito (effect size/ES) ${ }^{24}$. 


\section{Resultados}

As respostas da flexibilidade, da resistência muscular e do tempo para realização das tarefas funcionais são apresentadas na Figura 1. Interação $(F=7,76$ e $p<0,01)$ foi encontrada para a flexibilidade de ombro, com melhora para a SEQ B (pré $=-13,4 \pm 3,3 \mathrm{~cm}$ vs. pós $=-10,9 \pm 3,5$ $\mathrm{cm}, \mathrm{ES}=0,76$ [moderada magnitude]), quando comparado com a SEQ A (pré $=-14,7 \pm 3,3 \mathrm{~cm}$ vs. pós $=-17,4 \pm 3,5 \mathrm{~cm}, \mathrm{ES}=0,82$ [moderada magnitude]). Efeito do tempo para a tarefa funcional total (SEQ A $=-2,9 \%$ [ES = 0,50, pequena magnitude] e SEQ B $=-1,9 \%$ [ES $=0,30$, pequena magnitude], $\mathrm{F}=5,57$ e $p<0,05)$ e resistência muscular total (SEQ A $=+11,5 \%$ [ES $=3,00$, grande magnitude] e SEQ B $=+8,9 \%$ [ES $=2,33$, grande magnitude], $\mathrm{F}=29,14$ e $p<0,01)$ foram observadas para ambas as sequências de TP.
Para análise da carga de treino (Figura 2), mesmo sendo observado um incremento de $54,0 \%$ ( $E S=4,31$, grande magnitude) para a SEQ A e de 74,3\% (ES = 5,40, grande magnitude) para a SEQ B (efeito do tempo, $\mathrm{F}=28,23$ e $p$ $<0,01)$, não foram identificados efeitos isolados do grupo $(\mathrm{F}=0,00$ e $p=1,00)$ ou interação $(\mathrm{F}=$ $0,52$ e $p=0,79)$.

$\mathrm{Na}$ figura 3 são apresentadas as modificações relativas individuais (responsividade ao treino) da carga de treino, da flexibilidade, da resistência muscular total e tarefa funcional total. Dos idosos submetidos ao TP, 100\% foram responsivos para a carga de treino (painel A). Para as medidas de flexibilidade (painel B), de resistência muscular total (painel C) e de tarefa funcional total (painel D) foram reveladas responsividades ao TP na ordem de 56\%, 94\% e 78\%, respectivamente. Com relação à ordem, $72 \%$ da SEQ A e 89\% da SEQ B foram responsivos ao TP.
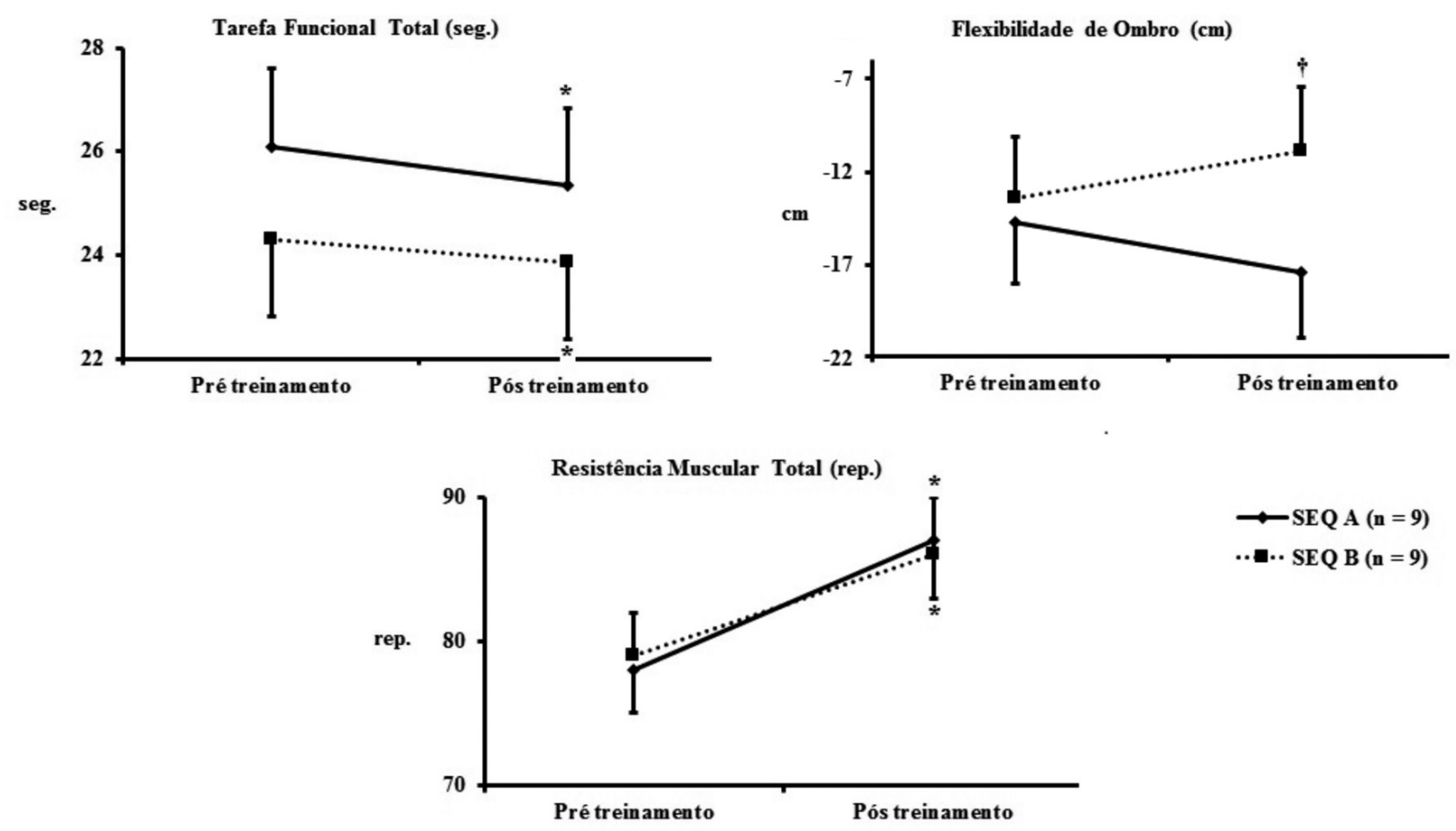

Figura 1: Resposta da tarefa funcional total, resistência muscular total e flexibilidade de ombro durante sete semanas de treinamento com pesos em idosos treinados $(\mathrm{n}=18)$, submetidos a duas sequências de exercícios com pesos (SEQ A e SEQ B)

Os valores estão expressos em média \pm desvio-padrão. * Diferença entre os momentos do experimento ( $p<$ $0,05)$. ${ }^{+}$Diferença entre as sequências de treinamento com pesos $(p<0,05)$.

Fonte: próprios autores. 


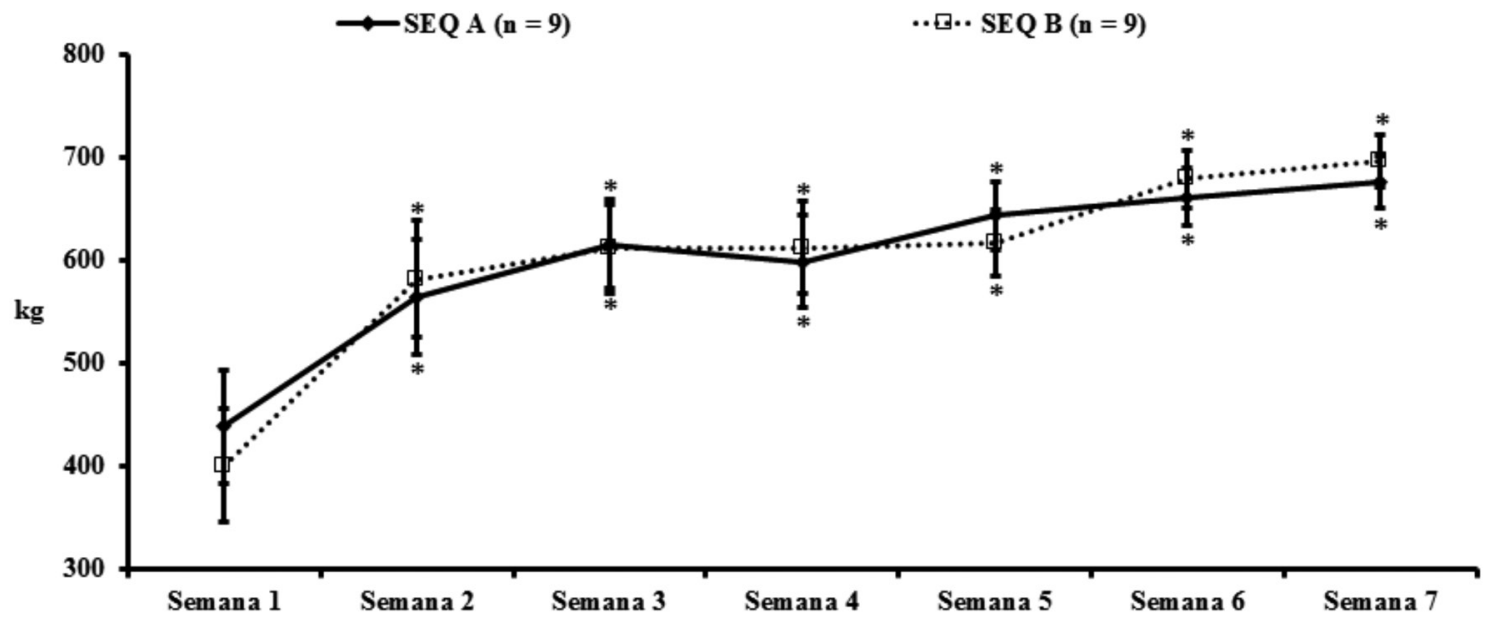

Figura 2: Carga de treino durante sete semanas de treinamento com pesos em idosos treinados $(n=18)$, submetidos a duas sequências de exercícios com pesos (SEQ A e SEQ B) Os valores estão expressos em média \pm desvio-padrão. ${ }^{*}$ Diferença entre as semanas de treinamento $(p<0,05)$. Fonte: próprios autores.
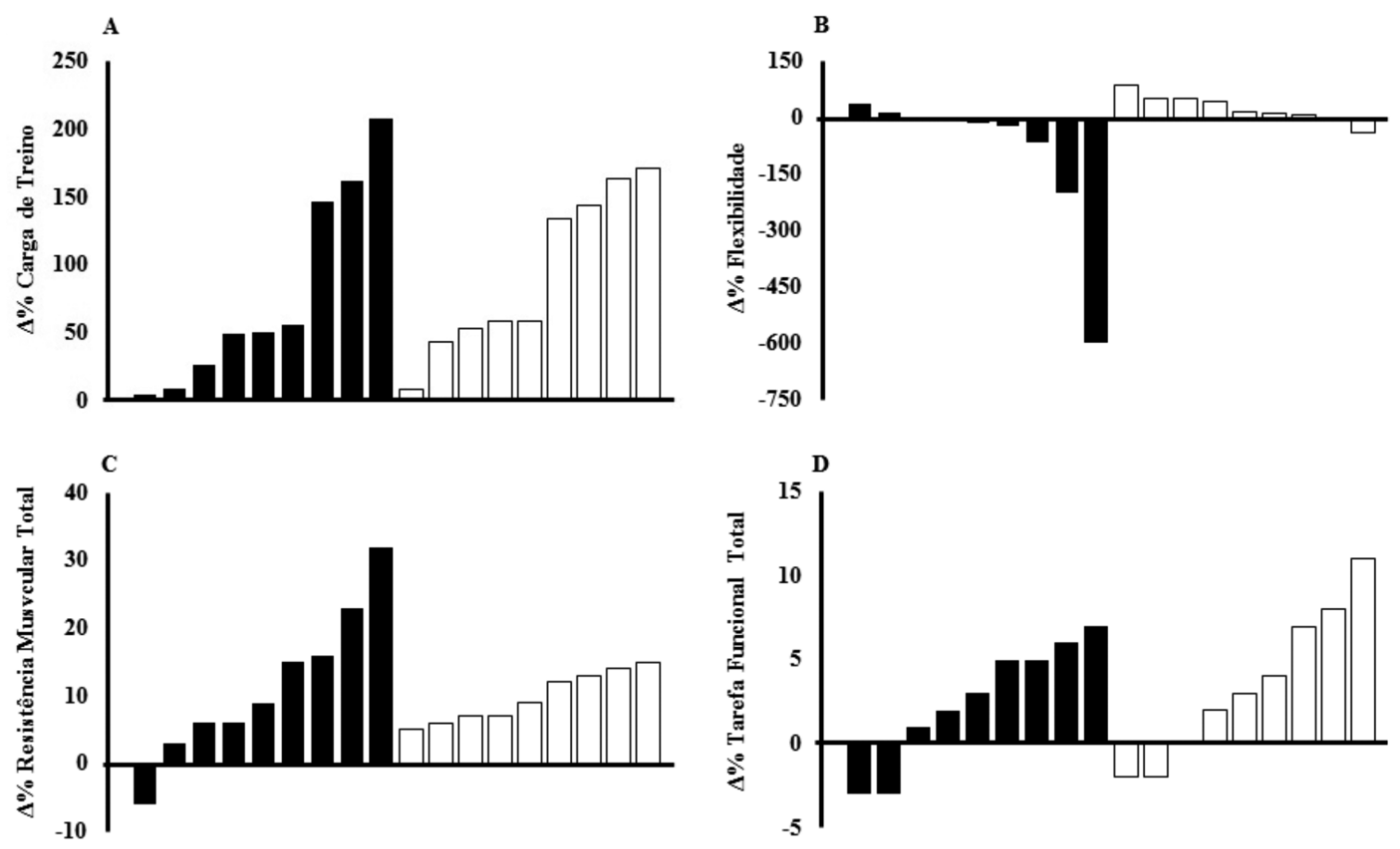

$\operatorname{SEQ} A(n=9)$

SEQ B (n = 9)

Figura 3: Modificações relativas individuais para a carga de treino (painel A), flexibilidade (painel B), resistência muscular total (painel C) e tarefa funcional total (painel D) após sete semanas de treinamento com pesos, em idosos treinados $(n=18)$, submetidos a duas sequências de exercícios com pesos (SEQ A e SEQ B).

Fonte: próprios autores. 


\section{Discussão}

Os principais resultados deste estudo foram: (1) o período de sete semanas de TP foi suficiente para desencadear mudanças positivas na tarefa funcional, resistência muscular e carga de treino, indiferente a ordem aplicada e (2) a SEQ B proporcionou melhora na flexibilidade. Os resultados confirmam que a prática regular de TP pode ser benéfica para a aptidão funcional em idosos treinados, proporcionandolhes maior proteção contra vários efeitos causados pelo processo de envelhecimento ${ }^{2}$, como o declínio na capacidade funcional. Os achados refutam a hipótese inicial e dos observados em outros trabalhos sobre ordem de $\mathrm{TP}^{13-15}$.

Para o conhecimento, este é o primeiro estudo que propôs comparar as respostas crônicas na capacidade funcional e carga de treino, a partir da manipulação na ordem de execução dos exercícios em idosos treinados, o que dificulta a comparação detalhada com a literatura para esta população. Estudos anteriores também relataram nenhuma diferença com relação à manipulação na ordem de execução dos exercícios em diferentes desfechos analisados para outras populações ${ }^{13-15,25}$.

Pirauá et al. ${ }^{25}$ observaram que a ordem não influenciou o volume total e a percepção subjetiva de esforço em homens jovens treinados. Já Dias et al. ${ }^{15}$ avaliaram o efeito da ordem dos exercícios na força muscular (teste de $1 \mathrm{RM}$ ), em homens jovens treinados, após 12 semanas de TP e observaram ganhos similares entre as diferentes ordens. Entretanto, Rodrigues et al. ${ }^{17}$ demonstraram que para mulheres idosas, a ordem do TP, em membros superiores, pode influenciar no número de repetições executadas. Embora o estudo de Rodrigues et al. ${ }^{17}$ tenha sido realizado com a população idosa, apresenta um protocolo transversal, o que dificulta as comparações.

Os achados de Rodrigues et al. ${ }^{17}$ demonstram as observações do estudo clássico de Sforzo e Touey ${ }^{11}$, em que a fadiga gerada pela alteração na ordem dos exercícios pode modificar os resultados. Vale considerar que os apontamentos de
Sforzo e Touey ${ }^{11}$ sobre fadiga, os quais também foram observados em outros estudos transversais ${ }^{9,10}$, podem não ser extrapolados para desenhos de caráter longitudinal. No presente trabalho ocorreu incremento da carga de treino, ao longo das semanas, refutando Sforzo e Touey ${ }^{11}$, em que a execução dos pequenos grupos musculares no início da sessão de TP não atrapalhou a continuidade do treino. Conforme apontado por Silva et al. ${ }^{19}$, o TP causa efeitos positivos em indivíduos idosos, desde que a duração do treinamento (tempo em semanas) seja suficientemente longa, independentemente da combinação de outras variáveis de treinamento (ordem, frequência, recuperação, número de séries, etc).

Outra questão, que deve ser considerada no presente experimento, foram as respostas positivas na capacidade funcional. Tais indicativos confirmam que o TP melhora a capacidade funcional de idosos ${ }^{5}$. Gerage et al. ${ }^{5}$ também verificaram, após doze semanas de um programa de TP, em idosas não treinadas, melhoras na capacidade funcional. Dado que a força é um fator para manutenção da mobilidade e funcionalidade desses indivíduos ${ }^{5}$ e, uma vez que a carga de treino representa uma medida de força ${ }^{4}$, possivelmente, as modificações na capacidade funcional, observadas no presente estudo, podem estar relacionadas às modificações na força muscular induzidas pelo TP.

Embora não se tenha avaliado a força muscular máxima (1 RM) no presente estudo, a progressão da carga de treino, bem como a duração do protocolo sugere esta melhora, já que a literatura sustenta as modificações na força muscular após poucas semanas de $\mathrm{TP}^{19}$, estando relacionada com melhoras em algumas tarefas funcionais ${ }^{5}$. Além disso, as adaptações neurais provenientes do TP exercem papel essencial no aumento da força ${ }^{19}$, sendo responsáveis pela coordenação intermuscular e multiarticular, aumentando a efetividade do movimento o que pode afetar, positivamente, a capacidade funcional dos idosos. Vale destacar que as possíveis adaptações neurais provenientes do TP não foram avaliadas no presente estudo. 
Com relação à flexibilidade, sete semanas de TP na SEQ B foi suficiente para aumentar a flexibilidade em idosos treinados. Os aumentos na flexibilidade, após um programa de TP em idosos, têm sido relatados previamente na literatura ${ }^{4}$. Carneiro et al. ${ }^{4}$ recrutaram $33 \mathrm{mu}$ lheres idosas não treinadas para executarem 12 semanas de TP e observaram aumento significativo na amplitude de movimento para flexão do quadril e extensão cervical, com execução de exercícios, que enfatizava a aplicação dos grandes para os pequenos grupos musculares.

Vale considerar que os mecanismos exatos responsáveis pelo aumento da flexibilidade, após um programa de TP ainda não foram estabelecidas na literatura, e os resultados extraídos do presente experimento não fornecem uma visão mecanicista. A flexibilidade está relacionada com os elementos morfológicos, tais como: os músculos, os ossos e os tecidos conjuntivos $^{4}$. Em particular, músculo e fáscia são responsáveis por aproximadamente $41 \%$ da resistência aplicada a um movimento, sugerindo que modificações na força podem reduzir a tensão destes tecidos, traduzindo em maior amplitude de movimento ${ }^{4}$. Entretanto, esta hipótese requer estudos mais aprofundados.

Mesmo apresentando resultados positivos do TP, para idosos treinados, deve se ressaltar que algumas limitações não podem ser desprezadas, como a ausência de grupo controle puro (sem exercício físico), o qual apontaria a eficácia do programa de TP em idosos, bem como outros formatos de ordem. A realização de um período de treinamento mais longo poderia apresentar respostas diferentes das encontradas no presente experimento. O cuidado com a coleta das informações, bem como a efetividade do treinamento comprovada pela melhora positiva das variáveis, enfatiza que o TP pode promover diversos benefícios ao idoso, colaborando com a manutenção de sua independência funcional ${ }^{18}$.

Em termos de aplicação prática, os resultados sugerem que é importante estruturar os programas de $\mathrm{TP}$, de acordo com a necessida- de e o objetivo de cada praticante, visto que a ordem de execução dos exercícios (grandes para os pequenos grupamentos musculares ou vice-versa) não influencia os resultados para a carga de treino, tarefa funcional e resistência muscular. Porém, quando o propósito de treinamento for modificar a flexibilidade, é importante verificar qual ordem de TP será aplicada. Além disso, os resultados do presente estudo permitem dar aos profissionais, que estão na área, maior liberdade na prescrição de exercícios, na perspectiva de uma maior aderência por parte dos praticantes.

\section{Conclusão}

Os resultados sugerem que o treinamento com pesos é efetivo para a melhora da tarefa funcional, da resistência muscular e da carga de treino em homens idosos treinados, porém a ordem do treinamento pode ser determinante nas modificações da flexibilidade.

\section{Conflito de interesses}

Os autores declaram que não apresentam conflitos.

\section{Referências}

1. Garber CE, Blissmer B, Deschenes MR, Franklin BA, Lamonte MJ, Lee IM, et al. American College of Sports Medicine: Position Stand. Quantity and quality of exercise for developing and maintaining cardiorespiratory, musculoskeletal, and neuromotor fitness in apparently healthy adults: guidance for prescribing exercise. Med Sci Sports Exerc. 2011; 43(7): 1334-59.

2. Chodzko-Zajko WJ, Proctor DN, Fiatarone Singh MA, Minson CT, Nigg CR, Salem GJ, et al. American College of Sports Medicine: Position Stand. Exercise and physical activity for older adults. Med Sci Sports Exerc. 2009; 41(7): 1510-30. 
3. Farinatti PT, Geraldes AA, Bottaro MF, Lima MV, Albuquerque RB, Fleck SJ. Effects of different resistance training frequencies on the muscle strength and functional performance of active women older than 60 years. J Strength Cond Res. 2013; 27(8): 2225-34.

4. Carneiro NH, Ribeiro AS, Nascimento MA, Gobbo LA, Schoenfeld BJ, Achour Júnior A, et al. Effects of different resistance training frequencies on flexibility in older women. Clin Interv Aging. 2015; 10: 531-38.

5. Gerage AM, Januário RSB, Nascimento MA, Pina FLC, Cyrino ES. Impacto de 12 semanas de treinamento com pesos sobre a aptidão físico-funcional de mulheres idosas. Rev Bras Cineantropom Desempenho Hum. 2013; 15(2): 145-54.

6. Gonzalez AM, Mangine GT, Fragala MS, Stout JR, Beyer KS, Bohner JD, et al. Resistance training improves single leg stance performance in older adults. Aging Clin Exp Res. 2014; 26(1): 89-92.

7. Brito Ade F, de Oliveira CV, Brasileiro-Santos Mdo $S$, Santos Ada. Resistance exercise with different volumes: blood pressure response and forearm blood flow in the hypertensive elderly. Clin Interv Aging. 2014; 12(9): 2151-58.

8. American College of Sports Medicine. Position Stand: Progression models in resistance training for healthy adults. Med Sci Sports Exerc. 2009; 41(3): 687-708.

9. Simão R, de Salles BF, Figueiredo T, Dias I, Willardson JM. Exercise order in resistance training. Sports Med. 2012; 42(3): 251-65.

10. Silva NSL, Monteiro WD, Farinatti PTV. Influência da ordem dos exercícios sobre o número de repetições e percepção subjetiva do esforço em mulheres jovens e idosas. Rev Bras Med Esporte. 2009; 15(3): 219-23.

11. Sforzo GA, Touey PR. Manipulating exercise order affects muscular performance during a resistance exercise training session. J Strength Cond Res. 1996; 10(1): 833-38.

12. Farinatti PT V, da Silva NSL, Monteiro WD. Influence of exercise order on the number of repetitions, oxygen uptake, and rate of perceived exertion during strength training in younger and older women. J Strength Cond Res. 2013; 27(3): 776-85.

13. Simão R, Spineti J, de Salles BF, Oliveira LF, Matta $\mathrm{T}$, Miranda $\mathrm{F}$, et al. Influence of exercise order on maximum strength and muscle thickness in untrained men. J Sports Sci Med. 2010; 9(1): 1-7.
14. Spineti J, de Salles BF, Rhea MR, Lavigne D, Matta $\mathrm{T}$, Miranda F, et al. Influence of exercise order on maximum strength and muscle volume in nonlinear periodized resistance training. J Strength Cond Res. 2010; 24(11): 2962-69.

15. Dias I, de Salles BF, Novaes J, Costa PB, Simão R. Influence of exercise order on maximum strength in untrained young men. J Sci Med Sport. 2010; 13(1): 65-9.

16. Pina FLC, Nascimento MA, Januário RSB, Gerage AM, de Oliveira AR, Cyrino ES. Influência da ordem de exercícios com pesos sobre a composição corporal em homens idosos. Rev Educ Fis UEM. 2013; 24(3): 443-51.

17. Rodrigues EF, da Costa LL, Leite RD. Influência da ordem dos exercícios resistidos na percepção subjetiva de esforço e número de repetições em mulheres idosas. ConScientiae Saúde. 2016; 15(3): 361-69.

18. Gavasso WC, Beltrame V. Capacidade funcional e morbidades referidas: uma análise comparativa em idosos. Rev Bras Geriatr Gerontol. 2017; 20(3): 398-408.

19. Silva NL, Oliveira RB, Fleck SJ, Leon AC, Farinatti P. Influence of strength training variables on strength gains in adults over 55 years-old: a meta-analysis of dose-response relationships. J Sci Med Sport. 2014; 17(3): 337-44.

20. Picorelli AM, Pereira DS, Felício DC, Dos Anjos DM, Pereira DA, Dias R, et al. Adherence of older women with strength training and aerobic exercise. Clin Interv Aging. 2014; 14(9): 323-31.

21. Andreotti RA, Okuma SS. Validação de uma bateria de testes de atividades da vida diária para idosos fisicamente independentes. Rev Paul Educ Fís. 1999; 13(1): 46-66.

22. Rikli RE, Jones CJ. Development and validation of a functional fitness test for community-residing older adults. J Aging Phys Act. 1999; 7: 129-61.

23. Nelson ME, Rejeski WJ, Blair SN, Duncan PW, Judge JO, King AC, et al. Physical activity and public health in older adults: Recommendation from the American College of Sports Medicine and the American Heart Association. Med Sci Sports Exerc. 2007; 39(8): 1435-45.

24. Cohen J. Statistical power analysis for the behavioral sciences. (2nd Edition) Hillsdale: New Jersey, 1988.

25. Pirauá ALT, Beltrão NB, Lima Júnior DRAA, Queiroz GR, Souza JG, Melo BM, et al. Effect of exercise order on the resistance training perfomance during a circuit training session. Rev Bras Cineantropom Desempenho Hum. 2014; 16(3): 325-33. 